\title{
Transformation-Based Fuzzy Rule Interpolation Using Interval Type-2 Fuzzy Sets
}

\author{
Chengyuan Chen ${ }^{1}$ and Qiang Shen ${ }^{2, *}$ \\ 1 School of Electrical and Information Engineering, Chongqing University of Science and Technology, \\ Chongqing 401331, China; ccy@cqust.edu.cn \\ 2 Department of Computer Science, Institute of Mathematics, Physics and Computer Science, \\ Aberystwyth University, Aberystwyth SY23 3DB, UK \\ * Correspondence: qqs@aber.ac.uk; Tel: +44-1970-622424
}

Received: 18 June 2017; Accepted: 10 August 2017; Published: 15 August 2017

\begin{abstract}
In support of reasoning with sparse rule bases, fuzzy rule interpolation (FRI) offers a helpful inference mechanism for deriving an approximate conclusion when a given observation has no overlap with any rule in the existing rule base. One of the recent and popular FRI approaches is the scale and move transformation-based rule interpolation, known as T-FRI in the literature. It supports both interpolation and extrapolation with multiple multi-antecedent rules. However, the difficult problem of defining the precise-valued membership functions required in the representation of fuzzy rules, or of the observations, restricts its applications. Fortunately, this problem can be alleviated through the use of type-2 fuzzy sets, owing to the fact that the membership functions of such fuzzy sets are themselves fuzzy, providing a more flexible means of modelling. This paper therefore, extends the existing T-FRI approach using interval type-2 fuzzy sets, which covers the original T-FRI as its specific instance. The effectiveness of this extension is demonstrated by experimental investigations and, also, by a practical application in comparison to the state-of-the-art alternative approach developed using rough-fuzzy sets.
\end{abstract}

Keywords: fuzzy rule interpolation; interval type-2 fuzzy sets; transformation-based interpolation

\section{Introduction}

The compositional rule of inference [1] offers an effective mechanism to deal with fuzzy inference for dense rule bases. Briefly, a dense rule base is a rule base where the input universe of discourse is covered completely. Given such a rule base and an observation that is at least partially covered by the rule base, the conclusion can be inferred from certain rules that intersect with the observation. However, for cases where a fuzzy rule base contains "gaps" (i.e., the so-called sparse rule base as termed in [2]), if a given observation has no overlap with the antecedent values of any rule, conventional fuzzy inference methods will fail.

Fortunately, using fuzzy rule interpolation (FRI) [3,4], certain conclusions may still be obtained. Moreover, with the help of FRI techniques, the complexity of a rule base can be reduced by omitting fuzzy rules, which may be approximated from their neighbouring ones. Nevertheless, despite these advantages, the application of traditional FRI methods may lead to abnormal and invalid fuzzy conclusions. One particular issue is that the convexity of the derived fuzzy values is not guaranteed [5], but convexity is often a crucial requirement for fuzzy inference in order to attain improved interpretability of the results.

In order to overcome such drawbacks, a number of significant extensions to the original FRI methods has been proposed in the literature, including [6-9]. In particular, the scale and move transformation-based approach (T-FRI) [10,11] and its generalisation [12] can handle interpolation and extrapolation involving multiple fuzzy rules, with each rule consisting of multiple antecedents. 
Such work also guarantees the uniqueness, as well as the normality and convexity of the interpolated conclusion. This approach has recently been further enhanced with a backward interpolation technique to enable chained rule interpolation [13]; a dynamic learning method to support efficient use of previously interpolated results for subsequent inference [14]; an adaptive mechanism to facilitate automatic error corrections in interpolative inference $[15,16]$; an extended adaptive fuzzy rule interpolation approach for polygonal fuzzy sets $[17,18]$; and a system that works through similarity-based reasoning with interpolative conditions [19].

There is little work in the area of FRI that can handle uncertainty in fuzziness itself however. This is because all of the aforementioned approaches are implemented based on conventional fuzzy set representations, i.e., type-1 fuzzy sets [20]. Whilst membership functions play an important role in defining fuzzy sets, it is sometimes extremely difficult, if not impossible, to give precise definitions for such membership functions. More generally, there may be different types of uncertainty in fuzzy rule-based systems that need to be captured or modelled [21]: (1) the variables that are used in the antecedents and the consequences of rules may be indiscernible; (2) the meanings of the words may be vague because the same word can mean different things to different people; (3) an object can belong to a given set or conceptual class to a partial degree, but that degree may itself be uncertain; (4) the obtained rules may be inconsistent when individual views are obtained from a group of experts; (5) observations attainable by inexact knowledge may be noisy and therefore randomly distributed.

Most of these types of uncertainty can be difficult to deal with in order to determine the crisp membership functions for the fuzzy sets used. For instance, certain weather conditions are considered as cold by all people, but others may be considered as cold by only certain individuals. That is, different people may indicate different temperatures as their understanding of the concept cold. The membership functions for different people may therefore be very different, depending on their perception, preference, experience, etc. As another example, suppose that the concept of interest is eye contact [22], denoted by $x$ with $x$ belonging to the intensity range of $[0,10]$, where zero indicates no eye contact and 10 represents maximum eye contact. One of the terms that may characterise the amount of perceived eye contact is "some eye contact". Suppose that 10 people are surveyed, and they are asked to locate the distribution for some eye contact on the scale $0-10$. Surely, it would not be expected that the same, precisely-described results are obtained from 10 individuals. Instead, different membership functions to depict such a fuzzy concept will be provided. Figure 1 shows such an example. The question is how such diverse information about the underlying concept may be captured and expressed.

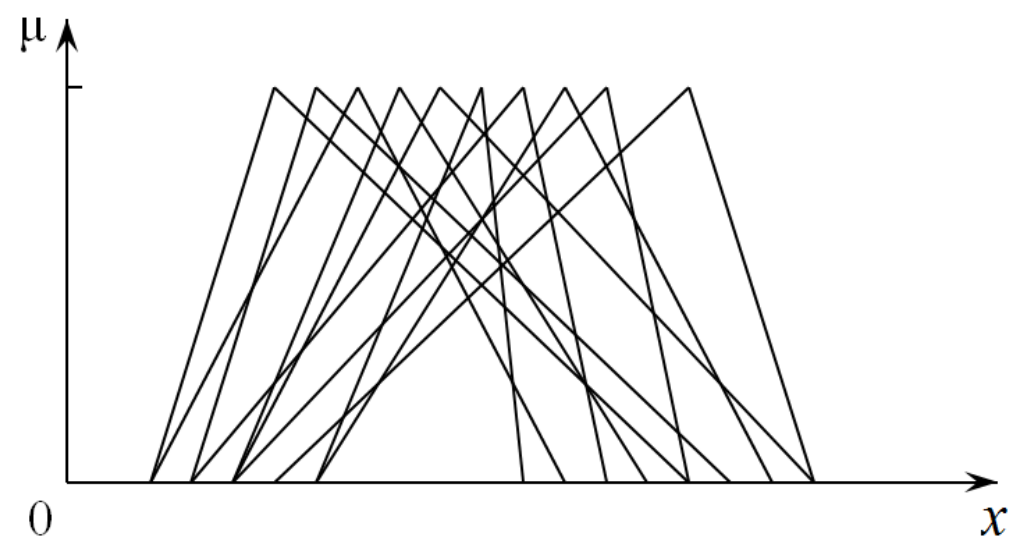

Figure 1. Different membership functions for some eye contact perceived by different people [23].

One approach might be to construct a type-1 fuzzy set with a triangle-shaped membership function whose support or base endpoints (on the $x$-axis) are the two average endpoint values (over the 10 surveyed results) and whose apex is midway between these two endpoints. However, this approach 
completely ignores the uncertainty associated with the different results. A second approach is to make use of the average endpoint values and the standard deviation of each endpoint to establish an uncertainty interval about each average endpoint value. In so doing, for each $x$, the membership of some eye contact is no longer a single value; instead, it is itself a function of the membership values. Such a concept has, therefore, to be represented as a type-2 fuzzy set [24], which is illustrated in Figure 2, where two endpoints $l$ and $r$ have two uncertainty intervals associated with them. The apex point $n$ is assumed to have a full certainty value. However, in general, $n$ can also have an uncertainty interval associated with it, just as with $l$ and $r$.

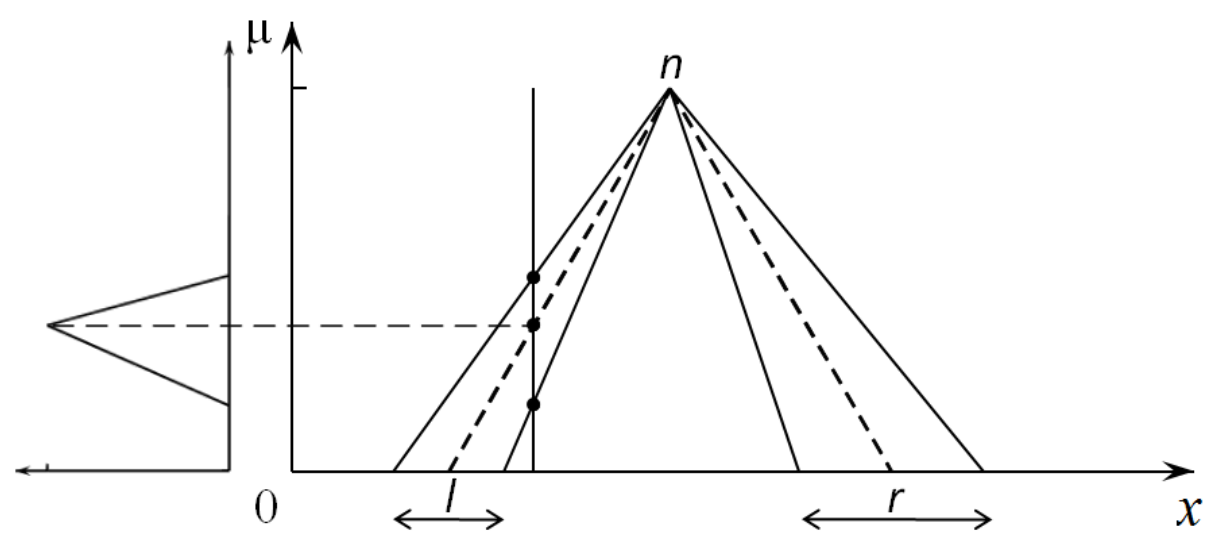

Figure 2. A type-2 fuzzy set corresponding to the situation depicted by Figure 1.

The concept of type-2 fuzzy sets was proposed as an extension of the concept of type- 1 fuzzy sets. A type-1 fuzzy set has a grade of membership that is crisp, whereas a type-2 fuzzy set has grades of membership that are themselves fuzzy $[25,26]$. That is, in set representation, membership functions of type-1 fuzzy sets are two-dimensional, whereas membership functions of type- 2 fuzzy sets are three-dimensional. It is the new third dimension that provides additional degrees of freedom for handling uncertainty [22]. Such sets are useful in circumstances where it is difficult to determine the exact and precise membership functions [27]. Note that apart from the use of type-2 fuzzy sets, other extensions have also been developed to represent various types of uncertainty that are associated with defining type-1 fuzzy sets, including interval-valued fuzzy sets [28,29], R-fuzzy sets [30], fuzzy complex numbers [31] and rough-fuzzy sets [23].

Type-2 fuzzy sets also include a specific group of fuzzy sets that are referred to as interval type-2 fuzzy sets [32,33], where all of the values of the secondary membership grades are the same. In this case, the third dimension is no longer needed because it conveys no new information. Although general type-2 fuzzy sets have one extra degree of freedom than interval type-2 fuzzy sets, it is not yet known how to best choose their secondary membership functions [34,35]. The third dimension is therefore often ignored in order to reduce the computational complexity, although this may result in the loss of an intuitively desirable degree of freedom in concept representation.

Of particular interest are the very recent developments, e.g., [36-38] that have been proposed to deal with FRI using interval type-2 fuzzy sets. However, none of these extend T-FRI. Motivated by this observation and based on the initial investigation of [39], this paper extends the existing T-FRI approach with the use of interval type- 2 fuzzy sets. The proposed approach not only facilitates the definition of representative values of interval type-2 fuzzy sets, but also modifies the underlying FRI technique to ensure intuitive interpolated conclusions.

The remainder of this paper is organised as follows. Section 2 reviews the general concepts of T-FRI and type-2 fuzzy sets. Section 3 presents the proposed extension to T-FRI, based on interval type-2 fuzzy sets. Case studies are provided to illustrate the interpolation results in Section 4. Conceptual comparisons between interval type-2 fuzzy sets and rough-fuzzy sets and, subsequently, a practical application comparison between the extensions respectively developed using these two types of 
higher-order fuzzy sets are presented in Section 5, noting that the extension to T-FRI using rough-fuzzy sets represents the start-of-the-art development in the relevant area. The paper is concluded in Section 6, including suggestions for possible further work.

\section{Background}

This section provides an overview of the basic concepts regarding T-FRI and type-2 fuzzy sets, to set the foundations for the subsequent development.

\subsection{Scale and Move Transformation-Based Interpolation}

An outline of the existing T-FRI is provided in this subsection; further details can be found in $[10,11]$. For simplicity, only rules involving triangle-shaped membership functions are considered in this paper.

Given a triangular fuzzy set $A$, denoted by $\left(a_{0}, a_{1}, a_{2}\right)$, where $a_{0}$ and $a_{2}$ are the left and right base points whose membership values equal zero, and $a_{1}$ is the normal point whose membership value equals one, its representative value (Rep) is defined as the average of the $x$ coordinates of these three key points, such that:

$$
\operatorname{Rep}(A)=\frac{a_{0}+a_{1}+a_{2}}{3}
$$

\subsubsection{N Closest Rules Selection}

Without losing generality, suppose that a rule $R_{i}$ and an observation $O$ are respectively represented by:

$$
\begin{aligned}
& R_{i}: \text { if } x_{1} \text { is } A_{i 1}, \cdots, x_{j} \text { is } A_{i j}, \cdots, x_{M} \text { is } A_{i M} \\
& \quad \text { then } y \text { is } B_{i} \\
& O: x_{1} \text { is } A_{1}^{*}, \cdots, x_{j} \text { is } A_{j}^{*}, \cdots, x_{M} \text { is } A_{M}^{*}
\end{aligned}
$$

where $A_{i j}$ denotes the $j$-th antecedent fuzzy set of rule $R_{i}, A_{j}^{*}$ denotes the observed fuzzy set of variable $x_{j}$ and $B_{i}$ denotes the consequent fuzzy set of rule $R_{i}$ with $j \in\{1, \ldots, M\}, M$ being the number of antecedent variables.

The distance $d_{i j}$ between the fuzzy set pair of $A_{i j}$ and $A_{j}^{*}$ for a given $j$ can be calculated as follows:

$$
d_{i j}=d\left(A_{i j}, A_{j}^{*}\right)=d\left(\operatorname{Rep}\left(A_{i j}\right), \operatorname{Rep}\left(A_{j}^{*}\right)\right)
$$

The distance $d_{i}$ between the rule $R_{i}$ and the observation $O$ is deemed to be the average of all antecedent variables' distances:

$$
d_{i}=\sqrt{\sum_{j=1}^{M} d_{i j}^{\prime 2}}, d_{i j}^{\prime}=\frac{d_{i j}}{\max _{j}-\min _{j}}
$$

where $\max _{j}$ and $\min _{j}$ are the maximum and minimum values of variable $j, j \in\{1, \ldots, M\}$, and each distance measure $d_{i j}$ is normalised into the range $[0,1]$, denoted by $d_{i j}^{\prime}$, to make the absolute distances compatible with each other over different variable domains.

\subsubsection{Intermediate Rule Construction}

Suppose $N(N \geq 2)$ closest rules have been chosen from the observation. Such rules are represented as $R_{i}, i \in\{1, \ldots, N\}$, and each has $M$ antecedents $A_{i j}, j \in\{1, \ldots, M\}$. Let $w_{A_{i j}}$ denote the weight to which the $j$-th antecedent of the $i$-th rule contributes to the intermediate rule. The normalised weight $w_{A_{i j}}^{\prime}$ can be defined as: 


$$
w_{A_{i j}}^{\prime}=\frac{w_{A_{i j}}}{\sum_{i=1}^{N} w_{A_{i j}}}, w_{A_{i j}}=\frac{1}{d_{i j}}
$$

Note that if $d_{i j}=0$, then $\operatorname{Rep}\left(A_{i j}\right)=\operatorname{Rep}\left(A_{j}^{*}\right)$. In this case, variable $j$ of the observation is considered to be "identical" to the corresponding antecedent of the rule $R_{i}$, in terms of the currently applied definition of Rep. Thus, in such exceptional cases, $w_{A_{i j}}=1$ for the "identical" variable(s), while $w_{A_{i j}}=0$ for the remainder.

The antecedent of the so-called intermediate fuzzy term $A_{j}^{I F T}$ is constructed from the antecedents of these closest rules. Another process shift is then introduced to modify $A_{j}^{I F T}$ to the antecedent of the intermediate rule $A_{j}^{\prime}$, so that it will have the same Rep as $A_{j}^{*}$ :

$$
A_{j}^{\prime}=A_{j}^{I F T}+\delta_{A_{j}}\left(\max _{j}-\min _{j}\right), \quad A_{j}^{I F T}=\sum_{i=1}^{N} w_{A_{i j}}^{\prime} A_{i j}
$$

where $\delta_{A_{j}}$ is a constant defined by:

$$
\delta_{A_{j}}=\frac{\operatorname{Rep}\left(A_{j}^{*}\right)-\operatorname{Rep}\left(A_{j}^{I F T}\right)}{\max _{j}-\min _{j}}
$$

Regarding the consequence of the intermediate rule $B^{\prime}$, it can be calculated by analogy to the computation of the rule antecedent, such that:

$$
B^{\prime}=B^{I F T}+\delta_{B}(\max -\min ), \quad B^{I F T}=\sum_{i=1}^{N} w_{B_{i}}^{\prime} B_{i}
$$

where $B^{I F T}$ is the consequence of the intermediate fuzzy term, max and min are the maximum and minimum values of the consequent variable and $w_{B_{i}}^{\prime}$ and $\delta_{B}$ are the means of $w_{A_{i j}}^{\prime}$ and $\delta_{A_{j}}$, $i \in\{1, \ldots, N\}, j \in\{1, \ldots, M\}$, respectively, which are defined as:

$$
w_{B_{i}}^{\prime}=\frac{1}{M} \sum_{j=1}^{M} w_{A_{i j^{\prime}}}^{\prime}, \delta_{B}=\frac{1}{M} \sum_{j=1}^{M} \delta_{A_{j}}
$$

\subsubsection{Scale Transformation}

The similarity degree between $\tilde{A}^{\prime}$ and $\tilde{A}^{*}$ is first measured by scale rate $s$, which is defined by:

$$
s=\frac{\tilde{a}_{2}^{*}-\tilde{a}_{0}^{*}}{\tilde{a}_{2}^{\prime}-\tilde{a}_{0}^{\prime}}
$$

Let $\tilde{A}^{\prime \prime}$ denote the modified intermediate term owing to the scale transformation. Using $s$, the current support $\left(\tilde{a}_{0}^{\prime}, \tilde{a}_{2}^{\prime}\right)$ is transformed into a new support $\left(\tilde{a}_{0}^{\prime \prime}, \tilde{a}_{2}^{\prime \prime}\right)$, while keeping the Rep and the ratio between the left-support $\left(\tilde{a}_{0}^{\prime \prime}, \tilde{a}_{1}^{\prime \prime}\right)$ and the right-support $\left(\tilde{a}_{1}^{\prime \prime}, \tilde{a}_{2}^{\prime \prime}\right)$ of $\tilde{A}^{\prime \prime}$ the same as those of its original. $\tilde{A}^{\prime \prime}$ can then be calculated by:

$$
\left\{\begin{array}{l}
\tilde{a}_{0}^{\prime \prime}=\frac{\tilde{a}_{0}^{\prime}(1+2 s)+\tilde{a}_{1}^{\prime}(1-s)+\tilde{a}_{2}^{\prime}(1-s)}{3} \\
\tilde{a}_{1}^{\prime \prime}=\frac{\tilde{a}_{0}^{\prime}(1-s)+\tilde{a}_{1}^{\prime}(1+2 s)+\tilde{a}_{2}^{\prime}(1-s)}{3} \\
\tilde{a}_{2}^{\prime \prime}=\frac{\tilde{a}_{0}^{\prime}(1-s)+\tilde{a}_{1}^{\prime}(1-s)+\tilde{a}_{2}^{\prime}(1+2 s)}{3}
\end{array}\right.
$$




\subsubsection{Move Transformation}

The similarity degree is further measured by move rate $m$. Using $m$, the current support $\left(\tilde{a}_{0}^{\prime \prime}, \tilde{a}_{2}^{\prime \prime}\right)$ of $\tilde{A}^{\prime \prime}$ is moved to $\left(\tilde{a}_{0}^{*}, \tilde{a}_{2}^{*}\right)$ while ensuring its Rep to be the same as that of the observation $\tilde{A}^{*}$. The move rate $m$ is defined by:

$$
m=\left\{\begin{array}{l}
\frac{\tilde{a}_{0}^{*}-\tilde{a}_{0}^{\prime \prime}}{\frac{\tilde{a}_{1}^{\prime \prime}-\tilde{a}_{0}^{\prime \prime}}{3}} \text { if } \tilde{a}_{0}^{*} \geq \tilde{a}_{0}^{\prime \prime} \\
\frac{\tilde{a}_{0}^{*}-\tilde{a}_{0}^{\prime \prime}}{\frac{\tilde{a}_{2}^{\prime \prime}-\tilde{a}_{1}^{\prime \prime}}{3}} \text { otherwise }
\end{array}\right.
$$

Given $m, \tilde{A}^{*}$ can then be calculated as:

$$
\left\{\begin{array}{l}
\left\{\begin{array}{l}
\tilde{a}_{0}^{*}=\tilde{a}_{0}^{\prime \prime}+m \frac{\tilde{a}_{1}^{\prime \prime}-\tilde{a}_{0}^{\prime \prime}}{3} \\
\tilde{a}_{1}^{*}=\tilde{a}_{1}^{\prime \prime}-2 m \frac{\tilde{a}_{1}^{\prime \prime}-\tilde{a}_{0}^{\prime \prime}}{3} \quad \text { if } m \geq 0 \\
\tilde{a}_{2}^{*}=\tilde{a}_{2}^{\prime \prime}+m \frac{\tilde{a}_{1}^{\prime \prime}-\tilde{a}_{0}^{\prime \prime}}{3}
\end{array}\right. \\
\left\{\begin{array}{l}
\tilde{a}_{0}^{*}=\tilde{a}_{0}^{\prime \prime}+m \frac{\tilde{a}_{2}^{\prime \prime}-\tilde{a}_{1}^{\prime \prime}}{3} \\
\tilde{a}_{1}^{*}=\tilde{a}_{1}^{\prime \prime}-2 m \frac{\tilde{a}_{2}^{\prime \prime}-\tilde{a}_{1}^{\prime \prime}}{3} \\
\tilde{a}_{2}^{*}=\tilde{a}_{2}^{\prime \prime}+m \frac{\tilde{a}_{2}^{\prime \prime}-\tilde{a}_{1}^{\prime \prime}}{3}
\end{array} \quad\right. \text { otherwise }
\end{array}\right.
$$

Note that the above scale and move transformations are utilised for one antecedent only. The calculation for $M$ antecedents is a mere repetition, resulting in $s_{j}$ and $m_{j}, j \in\{1, \ldots, M\}$.

The final interpolated conclusion $B^{*}$ is estimated by applying the scale and move transformations to $B^{\prime}$, using $s_{B}$ and $m_{B}$, such that:

$$
s_{B}=\frac{1}{M} \sum_{j=1}^{M} s_{j}, m_{B}=\frac{1}{M} \sum_{j=1}^{M} m_{j}
$$

where $M$ is the number of antecedent variables.

\subsection{Type-2 Fuzzy Sets}

A type-2 fuzzy set is characterised by a fuzzy membership function whose membership grade for each element is a fuzzy number in $[0,1]$. The formal definition is provided below.

Definition 1. [40] A type-2 fuzzy set, denoted $\tilde{A}$, is characterised by a type-2 membership function $\mu_{\tilde{A}}(x, u)$, where $x \in X$ and $u \in J_{x} \subseteq[0,1]$, i.e.,

$$
\tilde{A}=\left\{\left((x, u), \mu_{\tilde{A}}(x, u)\right) \mid \forall x \in X, \forall u \in J_{x} \subseteq[0,1]\right\}
$$

in which $0 \leq \mu_{\tilde{A}}(x, u) \leq 1$. $\tilde{\text { can }}$ also be expressed as:

$$
\tilde{A}=\int_{x \in X} \int_{u \in J_{x}} \mu_{\tilde{A}}(x, u) /(x, u) J_{x} \subseteq[0,1]
$$

where $\iint$ denotes union over all admissible $x$ and $u$.

Definition 2. [32] When all $\mu_{\tilde{A}}(x, u)=1$ then $\tilde{A}$ is an interval type-2 fuzzy set, which is expressed as:

$$
\tilde{A}=\int_{x \in X} \int_{u \in J_{x}} 1 /(x, u) J_{x} \subseteq[0,1]
$$


Definition 3. [32] Uncertainty in the primary memberships of an interval type-2 fuzzy set, $\tilde{A}$, consists of a bounded region that is called the footprint of uncertainty (FOU). It is the union of all primary memberships, i.e.,

$$
\operatorname{FOU}(\tilde{A})=\bigcup_{x \in X} J_{x}
$$

Definition 4. [32] The upper membership function (UMF), denoted $\tilde{A}^{U}$, and the lower membership function (LMF), denoted $\tilde{A}^{L}$, of a type-2 fuzzy set $\tilde{A}$ are two type-1 membership functions that bound the FOU. The UMF is associated with the upper bound of $F O U(\tilde{A})$ and is denoted $\mu_{\tilde{A}} u(x), \forall x \in X$, and the LMF is associated with the lower bound of $F O U(\tilde{A})$ and is denoted $\mu_{\tilde{A}^{L}}(x), \forall x \in X$, i.e.,

$$
\begin{array}{ll}
\mu_{\tilde{A}^{U}}(x)=\overline{\operatorname{FOU}(\tilde{A})} \quad \forall x \in X \\
\mu_{\tilde{A}^{L}}(x)=\underline{\operatorname{FOU}(\tilde{A})} \quad \forall x \in X
\end{array}
$$

As indicated above, an interval type-2 fuzzy set $\tilde{A}$ can be represented by the LMF $\tilde{A}^{L}$ and the $\mathrm{UMF} \tilde{A}^{U}$, i.e., $\tilde{A}=<\tilde{A}^{L}, \tilde{A}^{U}>$. In particular, when triangular membership functions are used, such an interval type-2 fuzzy set can be illustrated as shown in Figure 3 , where $\tilde{A}^{L}=\left(\tilde{a}_{0}^{L}, \tilde{a}_{1}^{L}, \tilde{a}_{2}^{L} ; H_{\tilde{A}^{L}}\right)$, $\tilde{A}^{U}=\left(\tilde{a}_{0}^{U}, \tilde{a}_{1}^{U}, \tilde{a}_{2}^{U} ; H_{\tilde{A}^{U}}\right),\left(\tilde{a}_{0}^{L}, \tilde{a}_{1}^{L}, \tilde{a}_{2}^{L}\right)$ and $\left(\tilde{a}_{0}^{U}, \tilde{a}_{1}^{U}, \tilde{a}_{2}^{U}\right)$ denote the three key points of the LMF and those of the UMF, respectively, and $H_{\tilde{A}^{L}}$ and $H_{\tilde{A}^{u}}$ denote the maximum membership values of $\tilde{A}^{L}$ and $\tilde{A}^{U}$, with $\tilde{a}_{0}^{U} \leq \tilde{a}_{0}^{L}, \tilde{a}_{2}^{L} \leq \tilde{a}_{2}^{U}, 0<H_{\tilde{A}^{L}} \leq H_{\tilde{A}^{U}}=1$. Clearly, the closer the shapes of $\tilde{A}^{L}$ and $\tilde{A}^{U}$ are, the less uncertain the information contained within $\tilde{A}$ is. When $\tilde{A}^{L}$ coincides with $\tilde{A}^{U}$, the interval type-2 fuzzy set degenerates to a type-1 fuzzy set.

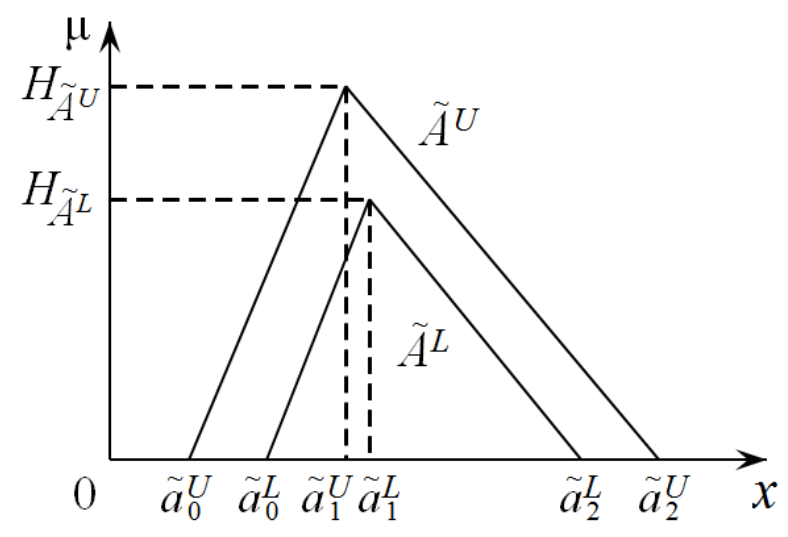

Figure 3. Lower membership function $\tilde{A}^{L}$ and upper membership function $\tilde{A}^{U}$ of a triangular interval type-2 fuzzy set $\tilde{A}$.

\section{Proposed Interval Type-2 Transformation-Based Interpolation}

Suppose that an interval type-2 fuzzy set $\tilde{A}$ as defined in Figure 3 has the following six distinct coordinates: $\left(\tilde{a}_{0}^{L}, 0\right),\left(\tilde{a}_{1}^{L}, H_{\tilde{A}}^{L}\right),\left(\tilde{a}_{2}^{L}, 0\right),\left(\tilde{a}_{0}^{U}, 0\right),\left(\tilde{a}_{1}^{U}, H_{\tilde{A}}^{U}\right)$ and $\left(\tilde{a}_{2}^{U}, 0\right)$. The lower and upper $\operatorname{Reps} \operatorname{Rep}\left(\tilde{A}^{L}\right)$ and $\operatorname{Rep}\left(\tilde{A}^{U}\right)$ of $\tilde{A}$ can then be computed according to Equation (1), such that:

$$
\left\{\begin{array}{l}
\operatorname{Rep}\left(\tilde{A}^{K}\right)_{x}=\frac{1}{3}\left(\tilde{a}_{0}^{K}+\tilde{a}_{1}^{K}+\tilde{a}_{2}^{K}\right) \\
\operatorname{Rep}\left(\tilde{A}^{K}\right)_{y}=\frac{1}{3} H_{\tilde{A}}^{K}
\end{array}\right.
$$

where $x$ and $y$ denote a certain variable and the corresponding membership function, respectively, $K \in\{L, U\}$.

Note that in the existing T-FRI, $\operatorname{Rep}(\tilde{A})_{y}$ is a constant, so only the $x$ coordinate value is therefore considered there. However, this is no longer the case in this work due to the introduction of additional 
uncertainty, and both values of the $x$ and $y$ coordinates need to be considered. The calculation for $\operatorname{Rep}(\tilde{A})_{y}$ follows the same mechanism as the one used to compute $\operatorname{Rep}(\tilde{A})_{x}$, to maintain consistency.

In order to distinguish different shapes of interval type-2 fuzzy sets, the shape diversity factor $f$ needs to be introduced. In this work, its specification follows the conventional definition of the statistical standard deviation (although this may be defined differently).

Definition 5. The lower and upper shape diversity factors $f_{\tilde{A}}^{L}$ and $f_{\tilde{A}}^{U}$ are defined by:

$$
f_{\tilde{A}}^{K}=\sqrt{\frac{\sum_{i=0}^{2}\left(\tilde{a}_{i}^{K}-\operatorname{Rep}\left(\tilde{A}^{K}\right)_{x}\right)^{2}}{3}}, K=L, U
$$

A small shape diversity factor implies that the four key points of $\tilde{A}^{L}\left(\tilde{A}^{U}\right)$ tend to be close to those of the lower (upper) Rep. That is, the smaller the shape diversity factor, the smaller the area of the LMF (UMF).

Mimicking the development of T-FRI where a Rep is used to succinctly depict a given type-1 fuzzy set, while extending T-FRI to dealing with interval type-2 fuzzy sets, a single overall Rep of a given interval type-2 fuzzy set is also introduced. For this, the weight factor $w$ of the LMF (UMF) is first defined as follows.

Definition 6. The lower and upper weight factors $w_{\tilde{A}}^{L}$ and $w_{\tilde{A}}^{U}$ are defined as the weights of the shape diversity factors, in terms of the areas of the LMF and UMF, such that:

$$
w_{\tilde{A}}^{K}=\frac{f_{\tilde{A}}^{K}}{f_{\tilde{A}}^{L}+f_{\tilde{A}}^{U}}, K=L, U
$$

where $f_{\tilde{A}}^{L}+f_{\tilde{A}}^{U} \neq 0$. If however, $f_{\tilde{A}}^{L}+f_{\tilde{A}}^{U}=0$, i.e., $f_{\tilde{A}}^{L}=0$ and $f_{\tilde{A}}^{U}=0$, the interval type-2 fuzzy set degenerates to a singleton value, $w_{\tilde{A}}^{L}=w_{\tilde{A}}^{U}=1 / 2$.

Definition 7. The overall representative value $\operatorname{Rep}(\tilde{A})$ of a given interval type-2 fuzzy set $\tilde{A}$ is defined by:

$$
\operatorname{Rep}(\tilde{A})=\sum_{K \in\{L, U\}}\left(w_{\tilde{A}}^{K} \sum_{e \in\{x, y\}} \operatorname{Rep}\left(\tilde{A}^{K}\right)_{e}\right)
$$

where the lower (upper) shape diversity factor is regarded as the weight of the lower (upper) Rep of the LMF (UMF). This is necessary, as otherwise, the same value for Rep would be derived from different shapes of interval type-2 fuzzy sets.

Given the above definitions, again, by mimicking the approach of the original T-FRI, the extended algorithm for deriving the interpolated conclusion with multiple multi-antecedent rules can be established and is summarised below.

1. Calculate representative values:

The lower and upper representative values $\operatorname{Rep}\left(\tilde{A}^{K}\right)_{x}$ and $\operatorname{Rep}\left(\tilde{A}^{K}\right)_{y}$ of a given interval type-2 fuzzy set $\tilde{A}$ are calculated first using Equation (19). The shape diversity factors $f_{\tilde{A}}^{K}$ and weight factors $w_{\tilde{A}}^{K}$ are computed according to Equations (20) and (21), respectively. The overall Rep $\operatorname{Rep}(\tilde{A})$ is then obtained by Equation (22), $K=L, U$. The calculations for all of the antecedent variables of all rules and their counterparts in the observation follow the same procedure. 
2. Choose closest $N$ rules:

The distances between the observation and all rules in the rule base are calculated using Equations (2) and (3). The $N(N \geq 2)$ rules that have minimal distances are then chosen as the $N$ closest rules to perform interpolation.

3. Construct intermediate rule

The normalised weight $w_{\tilde{A}_{i j}}^{\prime}$ of the $j$-th antecedent of the $i$-th chosen rule, which is calculated by Equation (4), together with the parameter $\delta_{\tilde{A}_{j}}$, which is calculated by Equation (6), are used in Equation (5) to obtain the value of each antecedent variable $x_{j}$ within the intermediate rule $\tilde{A}_{j}^{\prime}, i \in\{1, \ldots, N\}, j \in\{1, \ldots, M\}$. From this, two parameters $w_{\tilde{B}_{i}}^{\prime}$ and $\delta_{\tilde{B}}$ are computed using Equation (8) and are then used to construct $\tilde{B}^{\prime}$ from Equation (7), resulting in the intermediate rule $\tilde{A}_{1}^{\prime} \wedge \cdots \tilde{A}_{j}^{\prime} \wedge \cdots \tilde{A}_{M}^{\prime} \Rightarrow \tilde{B}^{\prime}$.

4. Perform scale, move and height transformations:

In conjunction with the given $\tilde{A}_{j}^{*}$ for each antecedent variable $x_{j}$, the rates $s_{j}^{K}, m_{j}^{K}$ and $h_{j}$, $K \in\{L, U\}$, can then be calculated using Equations (9), (11) and (23). Due to the extra uncertainty encountered in the membership functions, a further transformation on the height of the LMF is needed (because the LMFs of different interval type-2 fuzzy sets may have different heights), while the height of the UMF remains the same owing to its normality. This additional transformation is introduced to transform the heights of $\tilde{A}_{j}^{\prime L}$ to those of $\tilde{A}_{j}^{* L}$, with the height rate $h$ being calculated by:

$$
h_{j}=\frac{H_{\tilde{A}_{j}}^{* L}}{H_{\tilde{A}_{j}}^{\prime L}}
$$

where $0<H_{\tilde{A}_{j}}^{* L} \leq H_{\tilde{A}_{j}}^{* U}=1$ and $0<H_{\tilde{A}_{j}}^{\prime L} \leq H_{\tilde{A}_{j}}^{\prime} U$, as defined previously. This constraint applies to the interpolated conclusion, as well. That is, if the height of $\tilde{B}^{* L}$ is greater than the height of $\tilde{B}^{* U}$ after the height transformation, then $H_{\tilde{B}}^{* L}=H_{\tilde{B}}^{* U}$.

5. Derive interpolated conclusion:

The second intermediate term $\tilde{B}^{\prime \prime}$ and the interpolated result $\tilde{B}^{*}$ can then be estimated by the combined $s_{\tilde{B}}^{K}, m_{\tilde{B}}^{K}$ and $h_{\tilde{B}}, K \in\{L, U\}$. Here, $s_{\tilde{B}}^{K}$ and $m_{\tilde{B}}^{K}$ are computed following Equations (9)-(13), respectively, and $h_{\tilde{B}}$ is computed according to Equation (23) such that:

$$
h_{\tilde{B}}=\frac{1}{M} \sum_{j=1}^{M} h_{j}
$$

6. Implement modified procedure:

To obtain intuitive interpolated conclusions for interval type-2 fuzzy sets, the relative location between the LA and UA of an interval type-2 fuzzy set should be considered [39]. For this purpose, $B^{\prime \prime}$ is modified into $B_{c}^{\prime \prime}$ to maintain the relative location both before and after the scale transformation. Here, a relative location factor $\theta$ is defined by:

$$
\theta=\frac{B^{\prime L}}{B^{\prime} U}=\frac{B^{\prime \prime L}}{B_{n}^{\prime \prime U}}=\frac{B_{n}^{\prime \prime L}}{B^{\prime \prime U}}
$$

where $B_{n}^{\prime \prime} L$ and $B_{n}^{\prime \prime} U$ denote the "new" terms, which are modified from the given $B^{\prime \prime} U$ and $B^{\prime \prime} L$, respectively, using the same $\theta$. The combined $B_{c}^{\prime \prime} L$ and $B_{c}^{\prime \prime} U$ of $B_{c}^{\prime \prime}$ are then computed as the mean of the corresponding two terms, such that:

$$
B_{c}^{\prime \prime K}=\frac{B^{\prime \prime} K+B_{n}^{\prime \prime K}}{2}, K=L, U
$$


Similarly, the final interpolated conclusion can also be modified from $B^{*}$ to $B_{c}^{*}$ using the same $\theta$ to maintain the relative location both before and after the move transformation.

\section{Experimentation and Discussion}

In this section, four case studies are used to illustrate the interpolation results where the observations fall into the rule base "gaps". However, in general, if the observations partially overlap with the rule antecedents and such matches are above a certain confidence level, no interpolation will be required (as conventional compositional rule of inference can then be applied). Otherwise, the interpolation method is applied.

\subsection{Case 1}

This example considers an extreme case of the proposed approach where each interval type-2 fuzzy set under consideration has identical normal points. The observation and the two rules associated with the single antecedent variable are described in Table 1.

Table 1. Involved interval type-2 fuzzy sets for Case 1.

\begin{tabular}{cc}
\hline Rule 1 & $\begin{array}{c}\tilde{A}_{1}=<(1,3.5,4 ; 1),(0,3.5,5 ; 1)> \\
\tilde{B}_{1}=<(1.5,2,3 ; 1),(0,2,5 ; 1)>\end{array}$ \\
\hline Rule 2 & $\begin{array}{c}\tilde{A}_{2}=<(12,13,13.5 ; 1),(11,13,14 ; 1)> \\
\tilde{B}_{2}=<(11,11.5,12 ; 1),(10,11.5,13 ; 1)>\end{array}$ \\
\hline Observation & $\tilde{A}^{*}=<(6.5,8,9.5 ; 1),(6,8,10 ; 1)>$ \\
\hline
\end{tabular}

The interpolated conclusion $\tilde{B}_{c}^{*}=<(6.41,6.70,8.05 ; 1),(5.45,6.70,9.61 ; 1)>$ is shown in Figure 4. It can be seen that the normal points of the interpolated result are identical also. This shows that the modified procedure [39] is an effective improvement for achieving intuitive (or, equivalently, avoiding counter-intuitive) interpolated conclusions.
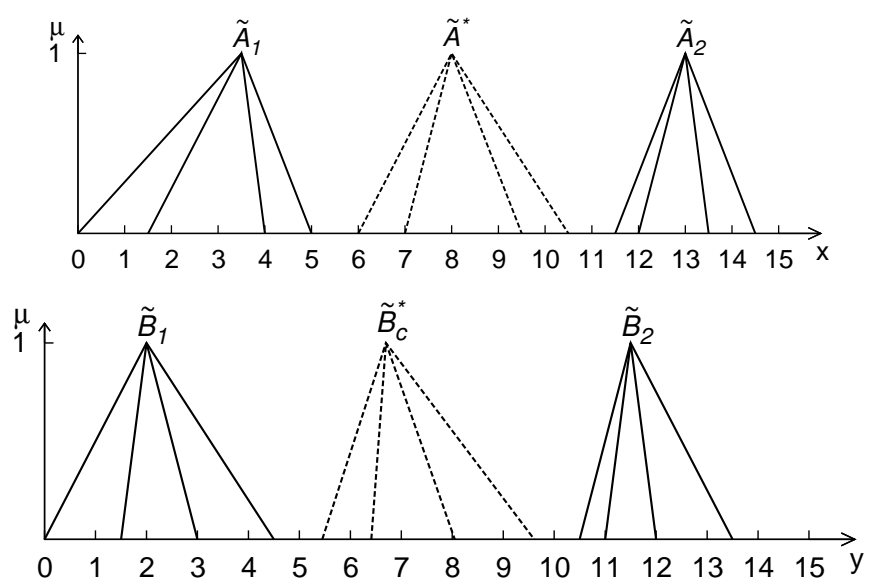

Figure 4. Two single-antecedent rules interpolation with identical normal points.

\subsection{Case 2}

This example case concerns an interpolation involving singleton-valued conditions using rules with multiple antecedent variables. In particular, two rules each involving two antecedents $\tilde{A}_{11} \wedge \tilde{A}_{12} \Rightarrow \tilde{B}_{1}, \tilde{A}_{21} \wedge \tilde{A}_{22} \Rightarrow \tilde{B}_{2}$ and the observation $\left\{\tilde{A}_{1}^{*}, \tilde{A}_{2}^{*}\right\}$ are given in order to illustrate the interpolative process in which the result $\tilde{B}_{c}^{*}$ is determined. All of the conditions are listed in Table 2. 
Table 2. Involved interval type-2 fuzzy sets for Case 2.

\begin{tabular}{c}
$\tilde{A}_{11}=<(3,3,3 ; 1),(3,3,3 ; 1)>$ \\
Rule $1 \quad \tilde{A}_{12}=<(2.5,2.5,2.5 ; 1),(2.5,2.5,2.5 ; 1)>$ \\
$\tilde{B}_{1}=<(4,4,4 ; 1),(4,4,4 ; 1)>$ \\
\hline Rule 2 $\quad \tilde{A}_{21}=<(12,13,13.5 ; 0.6),(11,13,14.5 ; 1)>$ \\
\\
$\tilde{A}_{22}=<(11.5,12.5,13.5 ; 0.5),(10,12,14.5 ; 1)>$ \\
$\tilde{B}_{2}=<(10.5,11.5,12 ; 0.5),(9,11.5,13 ; 1)>$ \\
Observation $\quad \tilde{A}_{1}^{*}=<(6,7,8 ; 0.6),(5,7.5,9 ; 1)>$ \\
$\tilde{A}_{2}^{*}=<(5.5,6,7 ; 0.5),(4,6,8 ; 1)>$
\end{tabular}

The interpolated conclusion $\tilde{B}_{c}^{*}=<(4.35,5.68,6.71 ; 0.54),(3.87,5.68,7.15 ; 1)>$ is shown in Figure 5. It follows that if certain components involved in the given rules are singleton-valued, the interpolated conclusion remains an interval type-2 fuzzy sets.
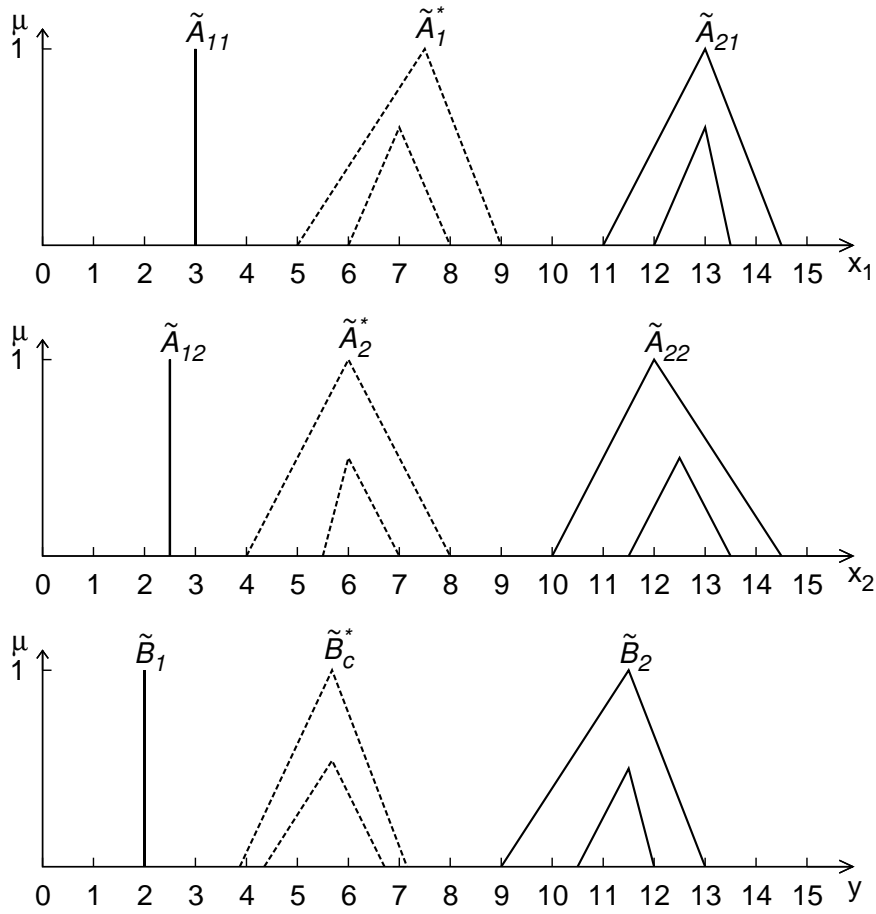

Figure 5. Two multi-antecedent rules interpolation with singleton-valued conditions.

\subsection{Case 3}

This example considers a general case involving multiple multi-antecedent rules. In particular, four rules with each involving three antecedent variables are selected as the closest neighbouring rules. Importantly, the values of two variables within the given observation are located between these rules, whereas one is located beyond the area flanked by them. This relates to a hybrid case, reflecting the needs for both interpolation and extrapolation [11]. All of the involved fuzzy sets are listed in Table 3.

The interpolated conclusion $\tilde{B}_{c}^{*}=<(7.52,8.72,9.77 ; 0.6),(6.61,8.86,10.71 ; 1)>$ is shown in Figure 6 . It can be seen that the interpolation and extrapolation hybrid performs well. 
Table 3. Hybridisation of interpolation and extrapolation for Case 3.

\begin{tabular}{|c|c|}
\hline Rule 1 & $\begin{array}{c}\tilde{A}_{11}=<(1,2,3 ; 0.7),(0,1.5,4 ; 1)> \\
\tilde{A}_{12}=<(2,3,4 ; 0.5),(1,2.5,5 ; 1)> \\
\tilde{A}_{13}=<(7,8,9 ; 0.6),(6,7.5,10 ; 1)> \\
\tilde{B}_{1}=<(1,1.5,2.5 ; 0.6),(0,1.5,3.5 ; 1)>\end{array}$ \\
\hline Rule 2 & $\begin{array}{c}\tilde{A}_{21}=<(16,17.5,19 ; 0.7),(15,17.5,20 ; 1)> \\
\tilde{A}_{22}=<(11.5,12.5,13 ; 0.5),(10,13,15 ; 1)> \\
\tilde{A}_{23}=<(21,22,23 ; 0.6),(20,22,23.5 ; 1)> \\
\tilde{B}_{2}=<(21.5,23,23.5 ; 0.6),(20.5,23,24 ; 1)>\end{array}$ \\
\hline Rule 3 & $\begin{array}{c}\tilde{A}_{31}=<(21.5,22.5,24 ; 0.7),(20,22,25 ; 1)> \\
\tilde{A}_{32}=<(6.5,7,8.5 ; 0.5),(5.5,7.5,9 ; 1)> \\
\tilde{A}_{33}=<(12,12.5,14 ; 0.6),(11,13,15 ; 1)> \\
\tilde{B}_{3}=<(16.5,17,18 ; 0.6),(15,17,20 ; 1)>\end{array}$ \\
\hline Rule 4 & $\begin{array}{l}\tilde{A}_{41}=<(11.5,12,13 ; 0.7),(10.5,12,14 ; 1)> \\
\tilde{A}_{42}=<(22,23,23.5 ; 0.5),(21,22.5,24 ; 1)> \\
\tilde{A}_{43}=<(17,18.5,19 ; 0.6),(16,18.5,19.5 ; 1)> \\
\tilde{B}_{4}=<(12,13,14 ; 0.6),(11.5,13.5,14.5 ; 1)>\end{array}$ \\
\hline Observation & $\begin{array}{c}\tilde{A}_{1}^{*}=<(6,6.5,7.5, ; 0.7),(5,6.5,9 ; 1)> \\
\tilde{A}_{2}^{*}=<(16.5,18,19.5 ; 0.5),(15,18,20 ; 1)> \\
\tilde{A}_{3}^{*}=<(1.5,2.5,4 ; 0.6),(0.5,3,5 ; 1)>\end{array}$ \\
\hline
\end{tabular}
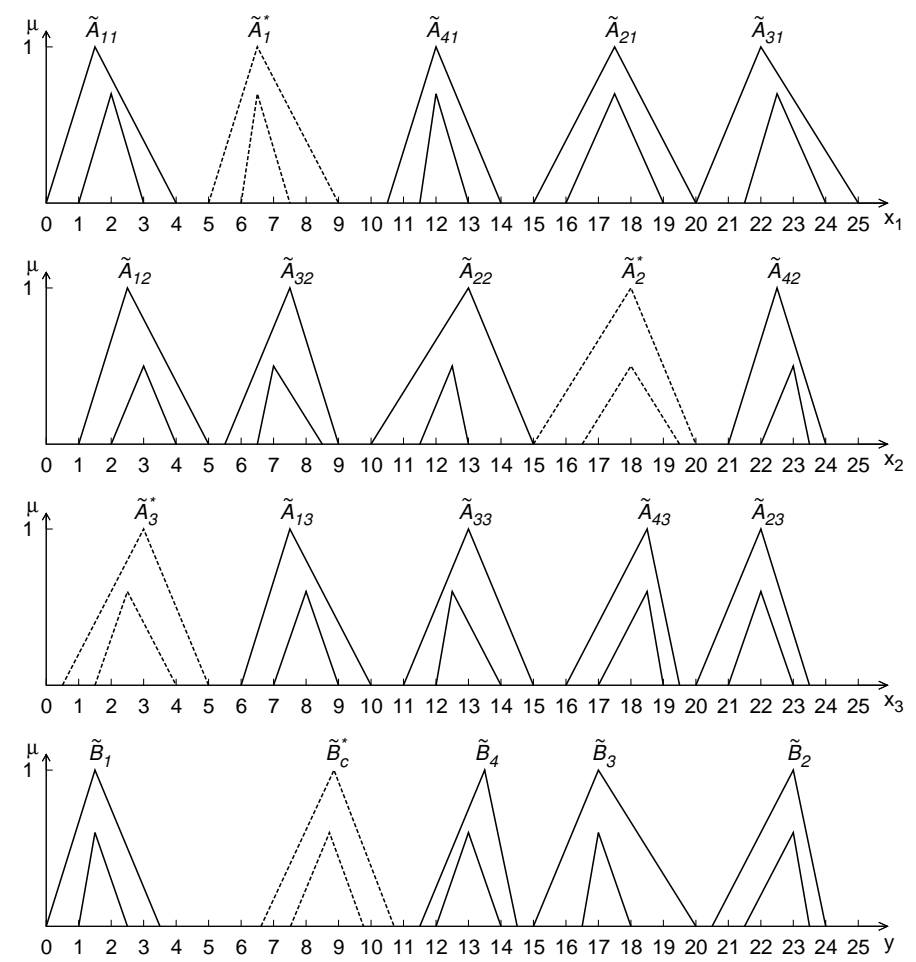

Figure 6. Interpolation and extrapolation involving multiple multi-antecedent rules for Case 3. 


\subsection{Case 4}

This example considers a specific case where all of the interval type-2 fuzzy sets degenerate to type- 1 fuzzy sets, i.e., $\tilde{A}_{i}^{L}=\tilde{A}_{i}^{U}$ and $\tilde{B}_{i}^{L}=\tilde{B}_{i}^{U}$. All of the terms are listed in Table 4 , where the type-1 fuzzy sets are still represented in the form of interval type-2 fuzzy sets.

Table 4. Involved interval type-2 fuzzy sets for Case 4.

\begin{tabular}{cc}
\hline \multirow{2}{*}{ Rule 1 } & $\tilde{A}_{1}=<(0,5,6 ; 1),(0,5,6 ; 1)>$ \\
& $\tilde{B}_{1}=<(0,2,4 ; 1),(0,2,4 ; 1)>$ \\
\hline \multirow{2}{*}{ Rule 2 } & $\tilde{A}_{2}=<(11,13,14 ; 1),(11,13,14 ; 1)>$ \\
& $\tilde{B}_{2}=<(10,11,13 ; 1),(10,11,13 ; 1)>$ \\
\hline Observation & $\tilde{A}^{*}=<(7,8,9 ; 1),(7,8,9 ; 1)>$ \\
\hline
\end{tabular}

The interpolated conclusion $\tilde{B}_{c}^{*}=<(5.83,6.26,7.38 ; 1),(5.83,6.26,7.38 ; 1)>$ is shown in Figure 7. The same result can be found in [10]. It follows that if all given sets are type-1 fuzzy sets, i.e., all uncertainty regarding the secondary membership function disappears, the interpolated result is the same as that achieved using the original T-FRI. This experimentally confirms that the extended version using interval type-2 fuzzy sets is a generalisation of the conventional T-FRI, covering T-FRI as its particular instance.
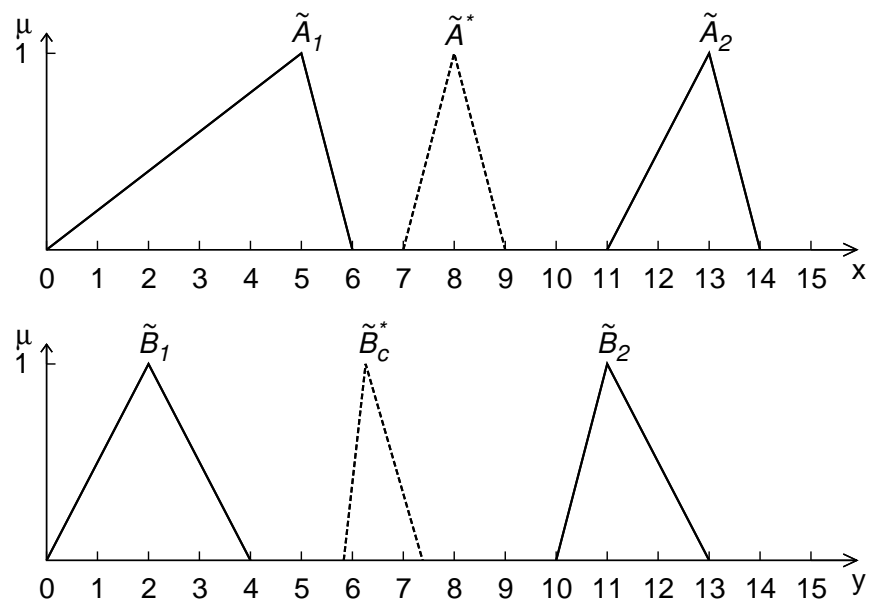

Figure 7. Interpolation for type-1 fuzzy sets case.

\section{Type-2 Fuzzy Sets vs. Rough-Fuzzy Sets for T-FRI}

As with type-2 fuzzy sets, rough-fuzzy sets [23] offer an alternative means for knowledge representation involving uncertainty, which are characterised by the lower and upper approximation membership functions. It is therefore not surprising that the present approach may be exploited to facilitate a closely relevant development of rough-fuzzy set-based T-FRI. Whilst such a development itself is beyond the scope of this paper, but reported elsewhere [23], it is interesting to examine the conceptual similarity between the two alternative techniques, thereby showing the generality of the underlying approach.

\subsection{Conceptual Comparison}

The uncertain information captured in the primary memberships of a general type-2 fuzzy set consists of a region FOU that is bounded by the lower and upper membership functions. However, 
for an interval type-2 fuzzy set, because the secondary membership grades convey no further information, the FOU is a complete description of all that is embedded in such a fuzzy set [32]. Thus, this single region is sufficiently used to describe uncertainty in interval type-2 fuzzy sets.

The concept of rough-fuzzy sets is derived from the definition of rough sets [41,42]. In particular, a rough-fuzzy set is constructed by the lower and upper approximation membership functions, where the lower approximation (LA) is defined by the intersection of all of the given fuzzy sets (certainty) and the upper approximation (UA) by the union of the given sets. That is, the LA captures the minimal level of certainty regarding the information embedded in the original fuzzy sets, and the UA reflects the maximal level. The boundary region, bounded by the two approximations, indicates the region that can possibly, but not always certainly, be partitioned with the information contained within the original fuzzy sets.

Comparing these two approaches to capturing higher-order uncertainty in describing given information content, it can be revealed that the UA in rough-fuzzy sets plays the same role as the interval in interval-valued fuzzy sets. In fact, the FOU in interval type- 2 fuzzy sets is the union of all primary memberships (intervals) [32]; therefore, it is also represented as an interval, covering all uncertain information involved. Both FOU and UA address all uncertain information. As the third-dimension of type-2 fuzzy sets becomes fixed with a membership value of 1 , the FOU in such an interval type-2 fuzzy set captures all uncertain information with full certainty, whilst such information can also be captured by means of the LA of a rough-fuzzy set. As a consequence, type- 2 fuzzy sets and rough-fuzzy sets are two different extensions of conventional fuzzy sets; the former is fuzzy-fuzzy sets, while the latter is rough-fuzzy sets. Nevertheless, the mathematical foundations of them are different, but they both can be utilised to represent knowledge involving uncertainty information, leading to the development of similar extensions to the conventional transformation-based fuzzy rule interpolation. From this point of view, the approach taken in this work is general, being minor in comparison to the methodology as reported in [23], where rough-fuzzy sets are utilised as the mathematical underpinning.

\subsection{Practical Application and Comparison}

Whilst conceptually it has been established that the present work using interval type-2 fuzzy sets to extend T-FRI bears a similar and general approach to that developed by the use of rough-fuzzy sets, this subsection investigates whether practically these techniques may function with similar results as well. To have a fair comparison, the same application problem as adopted in [23] is employed here, where the rough-fuzzy set-based extension to T-FRI is presented. The outline of the problem scenario is described below. Of course, this practical application will also serve as a further demonstration of the potential success of the proposed interval type-2 fuzzy set-based T-FRI approach.

\subsubsection{Application Problem}

Environmental change influences disease burden [43,44]. Intensive studies have been made in an effort to identify logical relationships underlying such influences so that the consequences of a certain environmental change may be predicted. One particular application problem in this area has recently been investigated in [15,45], which is based on the study of [46]. The original problem of [15] is simplified such that all of the examined factors are linearly connected. The resulting simpler causal model is shown in Figure 8.

All factors considered in this application study are represented as system variables, and each relation between two directly connected factors is represented as a rule associating the relevant variables. In summary, there are five variables in the problem: contact outside of the village, reintroduction of pathogenic strains, social connectedness, hygiene and sanitation infrastructure and infections disease rate, denoted as $x_{1}, \ldots, x_{5}$, respectively. Note that different variables are defined on different domains. To simplify knowledge representation, variable domains are mapped onto the real line and normalised. 


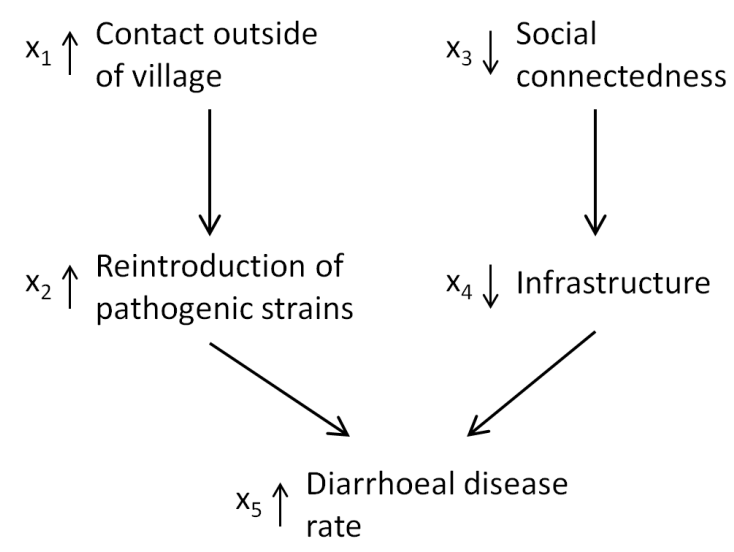

Figure 8. Causal diagram of the simplified application problem [23].

In order to evaluate the final disease rate, a group of experts is selected to express their views on each factor. As with the work of [23], suppose that the opinions are obtained from six experts, denoted as $T_{1}, \ldots, T_{6}$, respectively, and that the subsets of rules (one subset per causal implication): $A \rightarrow B$, $C \rightarrow D$ and $B \wedge D \rightarrow E$ are established by these experts with each supported by two of them. Note that here, $A, B, C, D$ and $E$ represent the certain qualitative values expressing the opinions given by the experts with regard to the domain variables $x_{1}, x_{2}, x_{3}, x_{4}$ and $x_{5}$, respectively.

\subsubsection{Results}

Given different expert rules and observations, one way to resolve the problem might be to use a conventional FRI approach, say T-FRI, to implement required interpolation separately. Suppose that two pairs of expert rules are contained in a sub-rule base: $A_{1} \rightarrow B_{1}$ and $A_{2} \rightarrow B_{2}$, where $A_{11} \rightarrow B_{11}$ and $A_{21} \rightarrow B_{21}$ are provided by the expert $T_{1}$ and $A_{12} \rightarrow B_{12}$ and $A_{22} \rightarrow B_{22}$ by $T_{2}$. Presented with two observations $A_{1}^{*}$ and $A_{2}^{*}$ (with the underlying semantics of the corresponding qualitative values depicted in Figure 9), the interpolated result by the use of T-FRI is a set that contains four elements. The computation with respect to the remainder of the subsets of rules follows the same procedure, resulting in a consequence set of 32 interpolated results, as shown in Figure 9. Obviously, the cardinality of the set of interpolated consequent results increases rapidly along with the increase of the cardinality of rule subsets and the number of observations. This results in high computational complexity.

Fortunately, the proposed interval type-2 approach can be applied without suffering from this difficulty. All of the uncertain relations can be captured using interval type-2 fuzzy sets, and the conclusion can be derived by the interval type- 2 fuzzy rule interpolation when a given observation is not matched with any of the rules available. The interpolated results following the present work are illustrated in Figure 10. These results reflect the distribution of those results shown in Figure 9. Compared to the rough-fuzzy-based interpolated results as given in Figure 11, which are directly taken from [23], it can be seen that the two resultant sets have similar locations. This implies that both approaches are effective in finding approximate solutions for this problem.

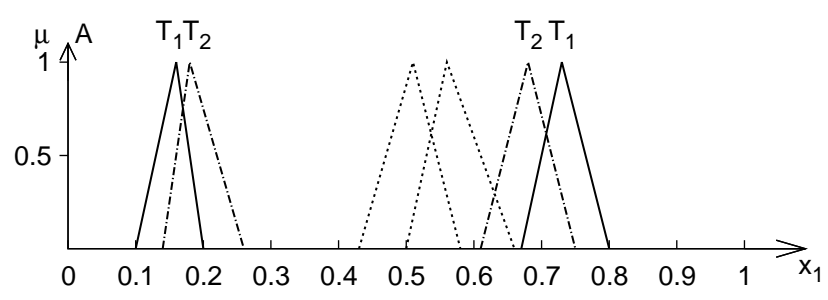

Figure 9. Cont. 

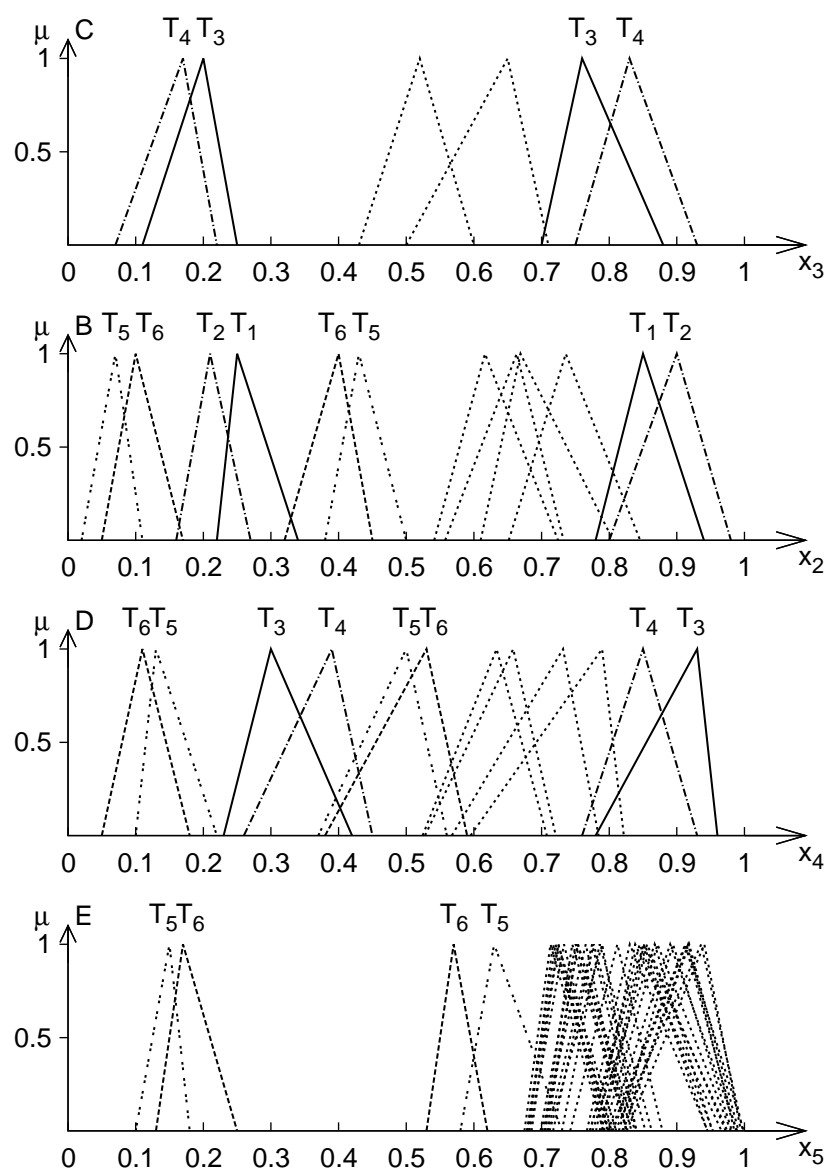

Figure 9. Interpolated results from conventional FRI [23].
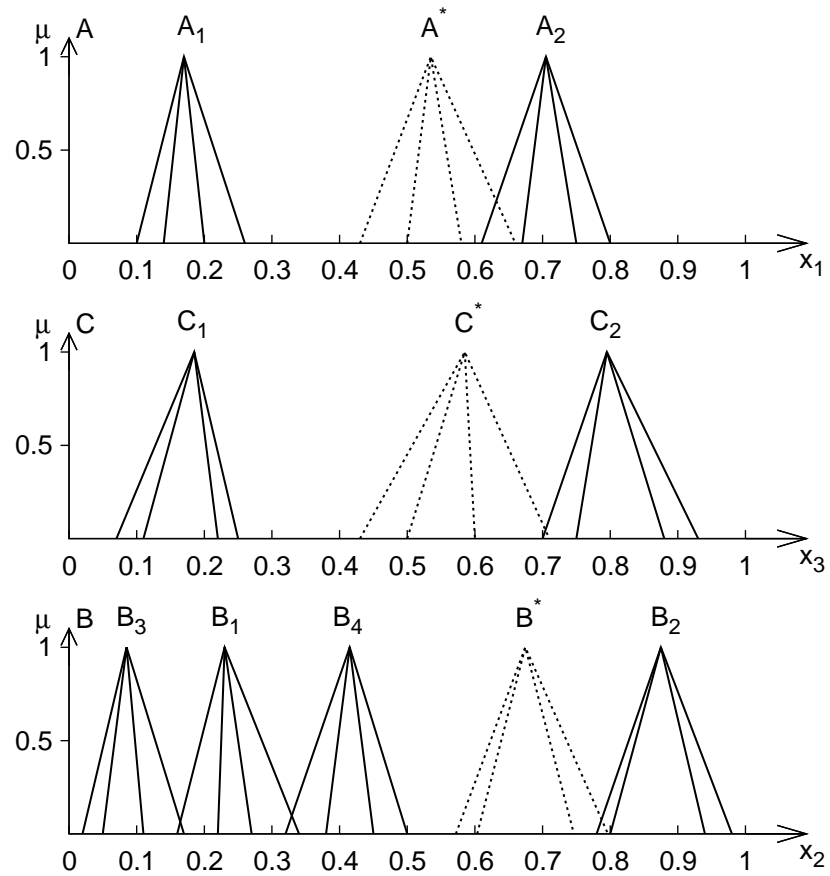

Figure 10. Cont. 

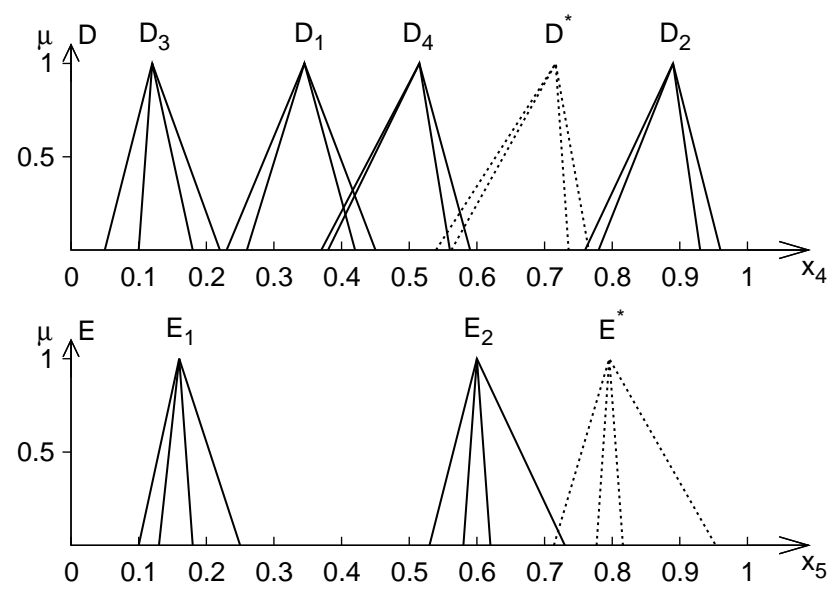

Figure 10. Interpolated results from interval type-2 interpolation [23].
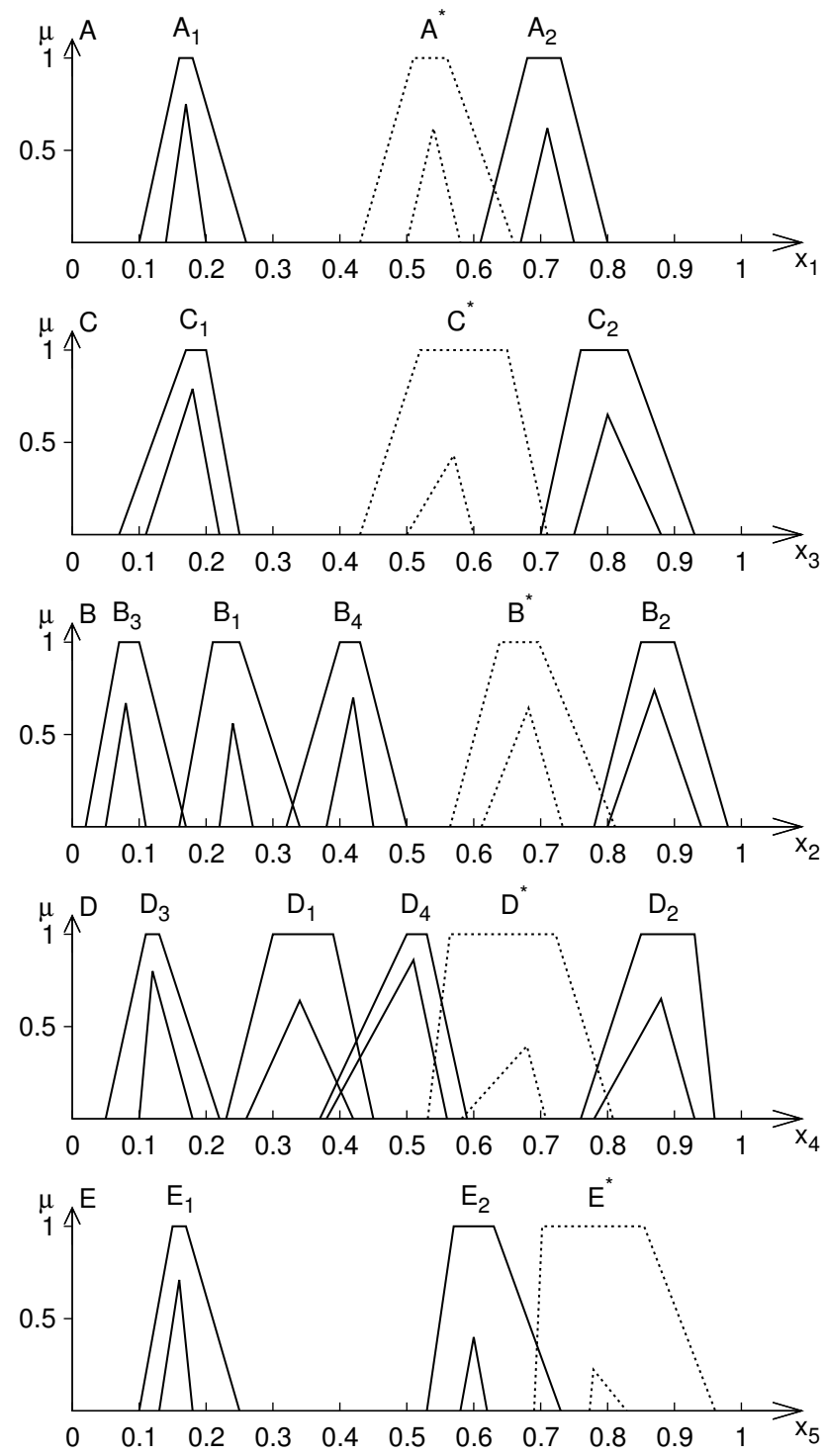

Figure 11. Interpolated results from rough-fuzzy interpolation (taken from [23]). 


\section{Conclusions}

This paper has proposed an extension to the existing transformation-based fuzzy rule interpolation approach through the use of interval type- 2 fuzzy sets. This approach offers a more concise way to deal with the uncertainty in fuzzy rule interpolation. Four demonstrative case studies have been presented in order to illustrate the operation of this novel approach. The conceptual and practical comparisons between type-2 fuzzy sets and rough-fuzzy sets have also been provided, with their implications to the extensions to T-FRI pointed out. The investigations have indicated that the proposed approach is of a natural appeal for FRI while dealing with the uncertainty that the conventional T-FRI technique may otherwise be difficult to handle. The work is an effective generalisation of the original T-FRI since the results of applying this extension degenerate to those obtainable by the existing type-1 FRI if the higher-order uncertainty embedded in the type- 2 fuzzy sets vanishes.

There are a number of aspects that could be improved with further research. In particular, only triangle-shaped membership functions are considered for implementation in this work. It would be beneficial to investigate how the approach may be extended (e.g., to enable the use of trapezoidal and other piecewise linear membership functions and to compare the effects of employing such different types of membership function). Furthermore, it would be interesting to evaluate the interpolated results attainable with this technique in comparison with those achievable using alternative FRI methods (e.g., [36-38]). Furthermore, currently, for simplicity (both mathematically and computationally), the current work is based on interval type-2 fuzzy sets. It would be useful to examine how the proposed work could be further generalised to support the involvement of general type-2 fuzzy sets while performing FRI. Finally, scaled-up real-world applications would help to further evaluate the full efficacy and potential of this research.

Acknowledgments: This research was partly supported by Aberystwyth University, UK, and partly by the Scientific and Technological Research Program of Chongqing Municipal Education Commission, China (Grant No. KJ1601316), and the Research Foundation of Chongqing University of Science and Technology, China (Grant No. CK2016B04).

Author Contributions: Chengyuan Chen and Qiang Shen conceived of and designed the experiments. Chengyuan Chen performed the experiments. Chengyuan Chen and Qiang Shen analysed the data. Chengyuan Chen and Qiang Shen wrote the paper.

Conflicts of Interest: The authors declare no conflict of interest. The founding sponsors had no role in the design of the study; in the collection, analyses or interpretation of data; in the writing of the manuscript; nor in the decision to publish the results.

\section{Abbreviations}

The following abbreviations are used in this manuscript:

FOU Footprint of uncertainty

FRI Fuzzy rule interpolation

LA Lower approximation

LMF Lower membership function

T-FRI Scale and move transformation-based fuzzy rule interpolation approach

UA Upper approximation

UMF Upper membership function

\section{References}

1. Zadeh, L.A. Outline of a new approach to the analysis of complex systems and decision processes. IEEE Trans. Syst. Man Cybern. 1973, 3, 28-44.

2. Wong, K.; Tikk, D.; Gedeon, T.; Kóczy, L. Fuzzy rule interpolation for multidimensional input spaces with applications: A case study. IEEE Trans. Fuzzy Syst. 2005, 13, 809-819.

3. Kóczy, L.; Hirota, K. Approximate reasoning by linear rule interpolation and general approximation. Int. J. Approx. Reason. 1993, 9, 197-225. 
4. Kóczy, L.; Hirota, K. Interpolative reasoning with insufficient evidence in sparse fuzzy rule bases. Inf. Sci. 1993, 71, 169-201.

5. Yan, S.; Mizumoto, M.; Qiao, W. Reasoning conditions on Kóczy's interpolative reasoning method in sparse fuzzy rule bases. Fuzzy Sets Syst. 1995, 75, 63-71.

6. Baranyi, P.; Kóczy, L.; Gedeon, T. A generalized concept for fuzzy rule interpolation. IEEE Trans. Fuzzy Syst. 2004, 12, 820-837.

7. Chang, Y.; Chen, S.; Liau, C. Fuzzy interpolative reasoning for sparse fuzzy-rule-based systems based on the areas of fuzzy sets. IEEE Trans. Fuzzy Syst. 2008, 16, 1285-1301.

8. Chen, S.; Ko, Y. Fuzzy interpolative reasoning for sparse fuzzy rule-based systems based on $\alpha$-cuts and transformations techniques. IEEE Trans. Fuzzy Syst. 2008, 16, 1626-1648.

9. Tikk, D.; Baranyi, P. Comprehensive analysis of a new fuzzy rule interpolation method. IEEE Trans. Fuzzy Syst. 2000, 8, 281-296.

10. Huang, Z.; Shen, Q. Fuzzy interpolative reasoning via scale and move transformations. IEEE Trans. Fuzzy Syst. 2006, 14, 340-359.

11. Huang, Z.; Shen, Q. Fuzzy interpolation and extrapolation: A practical approach. IEEE Trans. Fuzzy Syst. 2008, 16, 13-28.

12. Shen, Q.; Yang, L. Generalisation of scale and move transformation-based fuzzy interpolation. Adv. Comput. Intell. Intell. Inform. 2011, 15, 288-298.

13. Jin, S.; Diao, R.; Quek, C.; Shen, Q. Backward fuzzy rule interpolation. IEEE Trans. Fuzzy Syst. 2014, 22, 1682-1698.

14. Naik, N.; Diao, R.; Shen, Q. Dynamic fuzzy rule interpolation and its application to intrusion detection. In Proceedings of the 2016 IEEE International Conference on Fuzzy Systems (FUZZ-IEEE), Vancouver, BC, Canada, 24-29 July 2017.

15. Yang, L.; Shen, Q. Adaptive fuzzy interpolation. IEEE Trans. Fuzzy Syst. 2011, 19, 1107-1126.

16. Yang, L.; Chao, F.; Shen, Q. Generalised adaptive fuzzy rule interpolation. IEEE Trans. Fuzzy Syst. 2017, $25,4$.

17. Cheng, S.H.; Chen, S.M.; Chen, C.L. Adaptive fuzzy interpolation based on ranking values of polygonal fuzzy sets and similarity measures between polygonal fuzzy sets. Inf. Sci. 2016, 342, 176-190.

18. Chen, S.M.; Adam, S.I. Adaptive fuzzy interpolation based on general representative values of polygonal fuzzy sets and the shift and modification techniques. Inf. Sci. 2017, 414, 147-157.

19. Perfilieva, I. Closeness in similarity-based reasoning with an interpolation condition. Fuzzy Sets Syst. 2016, 292, 333-346.

20. Zadeh, L.A. Fuzzy sets. Inf. Control 1965, 8, 338-353.

21. Fu, X.; Shen, Q. Fuzzy compositional modeling. IEEE Trans. Fuzzy Syst. 2010, 18, 823-840.

22. Mendel, J. Type-2 fuzzy sets: Some questions and answers. IEEE Connect. Newsl. IEEE Neural Netw. Soc. 2003, 1, 10-13.

23. Chen, C.; Parthaláin, N.M.; Li, Y.; Price, C.; Quek, C.; Shen, Q. Rough-fuzzy rule interpolation. Inf. Sci. 2016, 351, 1-17.

24. Zadeh, L.A. The concept of a linguistic variable and its application to approximate reasoning-I. Inf. Sci. 1975, 8, 199-249.

25. Liang, Q.; Mendel, J. Interval type-2 fuzzy logic systems: Theory and design. IEEE Trans. Fuzzy Syst. 2000, 8, 535-550.

26. Wu, D. On the Fundamental Differences Between Interval Type-2 and Type-1 Fuzzy Logic Controllers. IEEE Trans. Fuzzy Syst. 2012, 20, 832-848.

27. Karnik, N.; Mendel, J.; Liang, Q. Type-2 fuzzy logic systems. IEEE Trans. Fuzzy Syst. 1999, 7, $643-658$.

28. Bustince, H. Indicator of inclusion grade for interval-valued fuzzy sets. Application to approximate reasoning based on interval-valued fuzzy sets. Int. J. Approx. Reason. 2000, 23, 137-209.

29. Dubois, D.; Prade, H. Interval-valued fuzzy sets, possibility theory and imprecise probability. In Proceedings of the International Conference in Fuzzy Logic and Technology, Barcelona, Spain, 7-9 September 2005.

30. Yang, Y.; Hinde, C. A new extension of fuzzy sets using rough sets: R-fuzzy sets. Inf. Sci. 2010, 180, 354-365.

31. Fu, X.; Shen, Q. Fuzzy complex numbers and their application for classifiers performance evaluation. Pat. Recognit. 2011, 44, 1403-1417.

32. Mendel, J.; John, R.; Liu, F. Interval type-2 fuzzy logic systems made simple. IEEE Trans. Fuzzy Syst. 2006, $14,808-821$. 
33. Wu, D. Approaches for Reducing the Computational Cost of Interval Type-2 Fuzzy Logic Systems: Overview and Comparisons. IEEE Trans. Fuzzy Syst. 2013, 21, 80-99.

34. Mendel, J.M.; Liu, X. Simplified Interval Type-2 Fuzzy Logic Systems. IEEE Trans. Fuzzy Syst. 2013, 21, 1056-1069.

35. Mendel, J. Type-2 fuzzy sets and systems: An overview. Comput. Intell. Mag. 2007, 2, $20-29$.

36. Chen, S.M.; Chang, Y.C.; Pan, J.S. Fuzzy Rules Interpolation for Sparse Fuzzy Rule-Based Systems Based on Interval Type-2 Gaussian Fuzzy Sets and Genetic Algorithms. IEEE Trans. Fuzzy Syst. 2013, 21, 412-425.

37. Chen, S.M.; Chen, Z.J. Weighted fuzzy interpolative reasoning for sparse fuzzy rule-based systems based on piecewise fuzzy entropies of fuzzy sets. Inf. Sci. 2016, 329, 503-523.

38. Chen, S.M.; Lee, L.W.; Shen, V.R. Weighted fuzzy interpolative reasoning systems based on interval type-2 fuzzy sets. Inf. Sci. 2013, 248, 15-30.

39. Chen, C.; Quek, C.; Shen, Q. Scale and move transformation-based fuzzy rule interpolation with interval type-2 fuzzy sets. In Proceedings of the IEEE International Conference on Fuzzy Systems, Hyderabad, India, 7-10 July 2013; pp. 1-8.

40. Mendel, J.; John, R. Type-2 fuzzy sets made simple. IEEE Trans. Fuzzy Syst. 2002, 10, 117-127.

41. Pawlak, Z. Rough sets. Int. J. Comput. Inf. Sci. 1982, 11, 341-356.

42. Pawlak, Z.; Grzymala-Busse, J.; Slowinski, R.; Ziarko, W. Rough sets. Commun. ACM 1995, 38, 88-95.

43. Colwell, R.R.; Epstein, P.R.; Gubler, D.; Maynard, N.; McMichael, A.J.; Patz, J.A.; Sack, R.B.; Shope, R. Climate and Human Health. Science 1998, 279, 968-969.

44. Morse, S.S. Factors in the emergence of infectious diseases. Emerg. Infect. Dis. 1995, 1, 7-15.

45. Yang, L.; Shen, Q. Closed form fuzzy interpolation. Fuzzy Sets Syst. 2013, 225, 1-22.

46. Eisenberg, J.N.; Cevallos, W.; Ponce, K.; Levy, K.; Bates, S.J.; Scott, J.C.; Hubbard, A.; Vieira, N.; Endara, P.; Espinel, M.; et al. Environmental change and infectious disease: How new roads affect the transmission of diarrheal pathogens in rural Ecuador. Proc. Natl. Acad. Sci. USA 2006, 103, 19460-19465.

(C) 2017 by the authors. Licensee MDPI, Basel, Switzerland. This article is an open access article distributed under the terms and conditions of the Creative Commons Attribution (CC BY) license (http:/ / creativecommons.org/licenses/by/4.0/). 